\title{
Editorial \\ A Special Issue on Modeling, Dimensioning, and Optimization of 5G Communication Networks, Resources, and Services
}

\author{
Ioannis D. Moscholios ${ }^{1, *(\mathbb{D}}$, Mariusz Głąbowski ${ }^{2}\left(\mathbb{D}\right.$, Panagiotis G. Sarigiannidis ${ }^{3}\left(\mathbb{D}\right.$ and Michael D. Logothetis ${ }^{4}(\mathbb{D})$ \\ 1 Department Informatics \& Telecommunications, University of Peloponnese, 22131 Tripolis, Greece \\ 2 Faculty of Computing and Telecommunications, Poznan University of Technology, 60965 Poznan, Poland; \\ mariusz.glabowski@put.poznan.pl \\ 3 Department Electrical and Computer Engineering, University of Western Macedonia, 50100 Kozani, Greece; \\ psarigiannidis@uowm.gr \\ 4 Department Electrical and Computer Engineering, University of Patras, 26504 Patras, Greece; \\ mlogo@upatras.gr \\ * Correspondence: idm@uop.gr
}

check for updates

Citation: Moscholios, I.D.; Głąbowski, M.; Sarigiannidis, P.G.; Logothetis, M.D. A Special Issue on Modeling, Dimensioning, and Optimization of 5G Communication Networks, Resources, and Services. Appl. Sci. 2022, 12, 1859. https:// doi.org/10.3390/app12041859

Received: 14 December 2021

Accepted: 31 January 2022

Published: 11 February 2022

Publisher's Note: MDPI stays neutral with regard to jurisdictional claims in published maps and institutional affiliations.

Copyright: (C) 2022 by the authors. Licensee MDPI, Basel, Switzerland. This article is an open access article distributed under the terms and conditions of the Creative Commons Attribution (CC BY) license (https:// creativecommons.org/licenses/by/ $4.0 /)$.

\section{Introduction}

Designing, dimensioning, and optimization of communication networks resources and services have been inseparable parts of the development of telecommunications since the very beginning of their existence. These networking problems have changed dramatically in recent years as a result of the changes in users' requirements for converging wired and wireless multiservice communications networks.

The global network of $5 \mathrm{G}$ remains a network of many heterogeneous systems interacting with each other, while widely used broadband mobile devices and cloud computing have given rise to tremendous growth in network traffic. Contemporary telecom networks must convey a large volume of traffic and provide service to traffic streams with highly differentiated requirements, in terms of bit rate and service time as well as required quality of both service and experience parameters. In such a communication infrastructure, there are important challenges, such as the study of necessary multilayer cooperation, new protocols, performance evaluation of different network parts, low layer network design, network management and security issues, and new technologies in general.

Each new type of network technology that is introduced in $5 \mathrm{G}$ is followed by a substantial increase in both the number and complexity of problems that need to be resolved by theoreticians and engineers. No matter what these developing changes may bring, the essential tasks for modern communication networks remain the same over the years-namely, (1) to develop new technologies offering increasing network capacity, (2) to determine and evaluate the relationship between the quality of service (quality of experience) parameters and the parameters characterizing traffic sources (services), (3) to control and optimize the usage of network resources, and (4) to enhance the capabilities of data transport, transmission, and reception between end users and the core network. These tasks provide a basis for developing engineering algorithms and tools used for designing, analysis, dimensioning, and optimization of wired and wireless transmission systems and networks.

\section{5G Communication Networks Modeling, Dimensioning, and Optimization}

Based on the above, this Special Issue aimed to amass state-of-the-art research contributions that address challenges in the design, dimensioning, and optimization of emerging $5 \mathrm{G}$ networks. After a rigorous review process, 16 papers were ultimately accepted for publication in this Special Issue, covering a range of important and well-timed subject areas.

The first paper, authored by I. Sousa, N. Sousa, M. Queluz, and A. Rodrigues, proposes a methodology for the evaluation and comparison of the performance of microwave radio transmission, free-space optics, and fiber optic technologies in the Cloud Radio Access 
Network (C-RAN) fronthaul segment, assuming different weather conditions, link lengths, and bit rate requirements [1]. Such a methodology enables the determination of the most cost-effective solution for each remote radio head (RRH)-baseband unit (BBU) link and the computation of the required number of BBUs. The second paper, authored by W. Lee, E. Suk Suh, W. Kwak, and H. Han, analyzes via simulation three different 5G communication network architectures-namely, the centralized cloud computing architecture, the multiaccess edge computing architecture, and the proposed Hybrid Cloud Computing (HCC) architecture, in terms of their response capability under normal and disruptive data traffic conditions [2]. The behavior of the abovementioned architectures was assessed using actual data traffic patterns obtained from the field, while the results obtained were used to quantify the advantages and disadvantages of each architecture and demonstrated the benefit of the new HCC architecture. The third paper, authored by K. Arshid, I. Hussain, M. Bashir, S. Naseem, A. Ditta, N. Mian, M. Zahid, and I. Khan, proposes a primary user traffic pattern-based opportunistic spectrum handoff (PUTPOSH) scheme for cognitive radio networks (CRNs) [3]. The proposed scheme permits a cognitive radio user to sense the primary user's traffic through energy-efficient sensing and then adopt a proactive or reactive handoff strategy according to the interarrival rate. Simulation results show that PUTPOSH maximizes the channel utilization and the throughput and, at the same time, minimizes the overall service time and the number of handoffs in CRNs. The fourth paper, authored by A. Alhammadi, M. Roslee, M. Alias, I. Shayea, and A. Alquhali, proposes a velocity-based self-optimization algorithm to adjust the handover control parameters in $4 \mathrm{G} / 5 \mathrm{G}$ networks [4]. The proposed algorithm utilizes the user's received power and speed to adjust the handover margin and the time to trigger during the user's mobility in the network. Based on simulation results, the authors show that the proposed self-optimization algorithm reduces the rate of ping-pong handovers and radio link failure, compared with other existing algorithms. The fifth paper, authored by Z. Zakrzewski, proposes a solution for enabling the coexistence of digitized radio-over-fiber and analog radio-over-fiber interfaces operating in the optical fronthaul of 5G mobile systems [5]. The sixth paper, authored by X. Li, C. Guo, J. Xu, L. Gupta, and R. Jain, studies the interesting subject of 5G network slices provisioning by taking into account both the slice node provisioning and the slice link provisioning aspects [6]. More specifically, the authors propose a two-stage heuristic slice provisioning algorithm for the $5 \mathrm{G}$ core network by jointly considering network resource attributes and topology attributes. Extensive simulation results show that the proposed algorithm increases the slice request acceptance ratio and, consequently, the revenue of the network infrastructure provider. The seventh paper, authored by M. Waqar and A. Kim, proposes and studies via simulation the end-to-end latency-aware path computation scheme, to improve the link utilization of capacity constraint Ethernet-based fronthaul bridged networks (EFBNs) in 5G C-RANs [7]. More specifically, the authors consider the queuing delays and end-to-end latencies for the selection of the optimal paths for individual enhanced Common Public Radio Interface (eCPRI) traffic streams in the EFBNs. In addition, they propose a packet forwarding mechanism that maximizes the transmissions of multiple eCPRI streams at tolerable latencies and improves the link distances between the RRHs and the BBU pool. The eighth paper, authored by M. Głabowski, A. Kaliszan, and M. Stasiak, proposes a method for the determination of the probability of strictly determined multiservice resources in a group of resources [8]. The proposed method allows the authors to elaborate a new formula for calculating the blocking probability of strictly determined resources in systems servicing multiservice traffic, e.g., in a group of cells in $4 \mathrm{G}$ and $5 \mathrm{G}$ systems. The proposed formula, whose accuracy is verified via simulation, is an extension of the well-known Palm-Jacobaeus loss formula elaborated for systems with single-rate traffic. The ninth paper, authored by P. Panagoulias, I. Moscholios, P. Sarigiannidis, M. Głabowski, and M. Logothetis, studies both analytically and through simulation the downlink of an orthogonal frequency division multiplexing based cell that services multirate traffic [9]. The call arrival process is random (Poisson) or quasi-random. To determine congestion probabilities and resource utilization, the cell is modeled as a multirate loss 
model, while the call admission is based on three different policies-restricted accessibility, bandwidth reservation, and complete sharing policies. In all three policies, recursive formulas are proposed for the determination of the various performance measures. The 10th paper, authored by Q. Zhu, W. Huang, K. Mao, W. Zhong, B. Hua, X. Chen, and Z. Zhao, proposes a discrete, non-stationary, multiple-input-multiple-output (MIMO) channel model suitable for the fixed-point realization on the field-programmable gate array (FPGA) hardware platform [10]. To this end, the authors develop a flexible hardware architecture with configurable channel parameters and implement it on a non-stationary MIMO channel emulator in a single FPGA chip. In addition, an improved non-stationary channel emulation method is employed to guarantee accurate channel fading and phase, and the schemes of other key modules are also illustrated and implemented in a single FPGA chip. Hardware tests presented by the authors show that the output statistical properties of the proposed MIMO channel emulator agree well with the corresponding theoretical ones. The 11th paper, authored by B. Seok, J. Sicato, T. Erzhena, C. Xuan, Y. Pan, and J. Park, studies and analyzes existing results about secure device-to-device (D2D) communication systems in terms of their security considerations and limitations [11]. In addition, a secure D2D communication system is proposed with the aim to address the security challenges and limitations of the existing results. The proposed secure D2D communication system was designed based on elliptic curve cryptography and lightweight authenticated encryption with associated data ciphers to cover resource-constrained IoT devices. The 12th paper, authored by K. Mohamed, M. Alias, and M. Roslee, studies the applicability of time division multiple access beamforming for future communication systems [12]. The authors propose the Sl-beamforming scheme for line-of-sight small cell systems in the LTE architecture. Simulation results show that the proposed scheme enhances the network signal to interference plus noise ratio and user average throughput, compared with nondominantly interfered region schemes. The 13th paper, authored by Z. Sun and Y. Zhao, proposes a novel time-varying least mean square adaptive filtering algorithm, with the aim to cancel the self-interference of near-end transceivers in co-frequency co-time full-duplex (CCFD) systems of the radio frequency domain [13]. The proposed algorithm solves the problem of strong self-interference of the radio frequency domain in CCFD systems by cooperatively controlling the autocorrelation values between the time factor and the error signal, to update the step size of the algorithm and provide a better quality of service for 5G communication networks. The 14th paper, authored by R. Aly, A. Zaki, W. Badawi, and $\mathrm{M}$. Aly, proposes a space-time, coded, orthogonal transform division multiplexing (STC OTDM) technique for 5G applications [14]. The proposed system enhances the data rate and performance of the orthogonal transform division multiplexing (OTDM) technique and is based on using space-time coding (STC) with OTDM, to increase the system diversity and consequently the system performance. Simulation results provided by the authors show that the proposed technique achieves better performance when compared with other multicarrier techniques. The 15th paper, authored by L. Wen, R. Razavi, and J. Lei, analyzes the intrinsic interference of filter bank-based multicarrier systems with isotropic orthogonal transfer algorithm pulse-shaping [15]. Such intrinsic interference is treated as a parity symbol and an iterative soft-in-soft-out detector, which is based on a message-passing algorithm, is proposed to exploit the useful information of the intrinsic interference. Finally, the last paper, authored by T. Tran, A. Ngoc, and N. Doan, presents an augmented Lagrangian-based alternating-direction inexact Newton (ALADIN) method in order to estimate the Laplacian spectrum in a decentralized scheme for dynamic controlled networks [16]. The key feature of this paper is a direct solution to non-convex optimization for Laplacian spectrum estimation using the proposed ALADIN method. 
Funding: This research received no external funding.

Acknowledgments: We would like to thank and congratulate all the authors for their valuable contributions to this Special Issue. We also thank the peer reviewers for their comments and suggestions, which helped the authors improve their papers. Finally, we would like to express our gratitude to the editorial team of MDPI Applied Sciences for their continuous support.

Conflicts of Interest: The authors declare no conflict of interest.

\section{References}

1. Sousa, I.; Sousa, N.; Queluz, M.P.; Rodrigues, A. Fronthaul Design for Wireless Networks. Appl. Sci. 2020, 10, 4754. [CrossRef]

2. Lee, W.; Suh, E.S.; Kwak, W.Y.; Han, H. Comparative Analysis of 5G Mobile Communication Network Architectures. Appl. Sci. 2020, 10, 2478. [CrossRef]

3. Arshid, K.; Hussain, I.; Bashir, M.K.; Naseem, S.; Ditta, A.; Mian, N.A.; Zahid, M.; Khan, I.A. Primary User Traffic Pattern Based Opportunistic Spectrum Handoff in Cognitive Radio Networks. Appl. Sci. 2020, 10, 1674. [CrossRef]

4. Alhammadi, A.; Roslee, M.; Alias, M.Y.; Shayea, I.; Alquhali, A. Velocity-Aware Handover Self-Optimization Management for Next Generation Networks. Appl. Sci. 2020, 10, 1354. [CrossRef]

5. Zakrzewski, Z. D-RoF and A-RoF Interfaces in an All-Optical Fronthaul of 5G Mobile Systems. Appl. Sci. 2020, 10, 1212. [CrossRef]

6. Li, X.; Guo, C.; Xu, J.; Gupta, L.; Jain, R. Towards Efficiently Provisioning 5G Core Network Slice Based on Resource and Topology Attributes. Appl. Sci. 2019, 9, 4361. [CrossRef]

7. Waqar, M.; Kim, A. Performance Improvement of Ethernet-Based Fronthaul Bridged Networks in 5G Cloud Radio Access Networks. Appl. Sci. 2019, 9, 2823. [CrossRef]

8. Głąbowski, M.; Kaliszan, A.; Stasiak, M. A Palm-Jacobaeus Loss Formula for Multi-Service Systems with Separated Resources. Appl. Sci. 2020, 10, 4019. [CrossRef]

9. Panagoulias, P.I.; Moscholios, I.D.; Sarigiannidis, P.G.; Głąbowski, M.; Logothetis, M.D. An Analytical Framework in OFDM Wireless Networks Servicing Random or Quasi-Random Traffic. Appl. Sci. 2019, 9, 5376. [CrossRef]

10. Zhu, Q.; Huang, W.; Mao, K.; Zhong, W.; Hua, B.; Chen, X.; Zhao, Z. A Flexible FPGA-Based Channel Emulator for Non-Stationary MIMO Fading Channels. Appl. Sci. 2020, 10, 4161. [CrossRef]

11. Seok, B.; Sicato, J.C.S.; Erzhena, T.; Xuan, C.; Pan, Y.; Park, J.H. Secure D2D Communication for 5G IoT Network Based on Lightweight Cryptography. Appl. Sci. 2020, 10, 217. [CrossRef]

12. Mohamed, K.S.; Alias, M.Y.; Roslee, M. Interference Avoidance Using TDMA-Beamforming in Location Aware Small Cell Systems. Appl. Sci. 2019, 9, 4979. [CrossRef]

13. Sun, Z.-Y.; Zhao, Y.-J. A Novel Self-Interference Cancellation Method Using an Optimized LMS Algorithm in CCFD Systems for a 5G Communication Network. Appl. Sci. 2019, 9, 3308. [CrossRef]

14. Aly, R.M.; Zaki, A.; Badawi, W.K.; Aly, M.H. Time Coding OTDM MIMO System Based on Singular Value Decomposition for 5G Applications. Appl. Sci. 2019, 9, 2691. [CrossRef]

15. Wen, L.; Razavi, R.; Lei, J. Intrinsic Interference Use for FBMC-IOTA Systems. Appl. Sci. 2019, 9, 3210. [CrossRef]

16. Tran, T.-M.-D.; Ngoc An, L.; Doan, N.C.N. Concensus-Based ALADIN Method to Faster the Decentralized Estimation of Laplacian Spectrum. Appl. Sci. 2020, 10, 5625. [CrossRef] 